\title{
Dinâmica espaço-temporal da paisagem e estrutura populacional de Euterpe precatoria Mart. em fragmento florestal no município mato-grossense de Alta Floresta, Brasil
}

\author{
Space-temporal dynamics of the landscape and population structure of Euterpe \\ precatoria Mart. in forest fragment in the municipality of Alta Floresta, Mato \\ Grosso state, Brazil
}

\section{Jakeline Santos Cochev ${ }^{\mathrm{I}}$, Ana Aparecida Bandini Rossi ${ }^{\mathrm{II}}$, Alex de Souza Rodrigues ${ }^{\mathrm{III}}$, Kelli Évelin Muller Zortea ${ }^{\mathrm{Iv}}$, Sandra Mara Alves da Silva Neves ${ }^{\mathrm{v}}$}

\begin{abstract}
Resumo
O Euterpe precatoria Mart., é utilizado na indústria de cosmético e alimentícia e vem sendo cada vez mais explorado no ambiente Amazônico. O objetivo deste trabalho é analisar a dinâmica espaço-temporal da paisagem e o efeito da fragmentação na estrutura e no padrão de distribuição espacial da espécie Euterpe precatoria, tendo como modelo um fragmento florestal no município de Alta Floresta-MT. Foram utilizadas imagens orbitais do satélite Landsat-2/MSS de 1975, Landsat-3/MSS de 1979, Landsat-5/TM de 1985, 1990, 1995, 2000, 2005 e 2010 e do Landsat-8/OLI de 2017. A partir dos dados gerados por meio de sensoriamento remoto foi calculado o Índice de Transformação Antrópica - ITA e Regressão Linear. Foram traçadas duas parcelas de $200 \mathrm{~m} \times 25 \mathrm{~m}$, no sentido borda-interior. A estrutura horizontal e vertical da espécie foi caracterizada por meio das análises: número de indivíduos, densidade, área basal, distribuição diamétrica e distribuição espacial. O padrão de distribuição espacial foi estimado pelo índice de Morisita, com um nível de significância de 5\%. As primeiras atividades antrópicas no entorno do fragmento de estudo iniciaramse a partir de $1984 \mathrm{com}$ a abertura da rodovia MT-325. A intensificação do uso da terra contribuiu para a redução da vegetação, cujo período mais crítico ocorreu entre 1990 a 2000. Foram amostrados 102 indivíduos na parcela A e 218 na parcela B. A densidade foi maior na parcela B. As duas parcelas apresentaram maior número de indivíduos distribuídos na primeira classe diamétrica. A população analisada apresentou dois padrões de distribuição espacial, padrão aleatório na parcela A e padrão agregado na parcela B. Euterpe precatoria encontra-se balanceado quanto ao processo de sucessão e recrutamento dos indivíduos, pois os valores da razão 'q' mostram que há um grande número de indivíduos nas primeiras classes de altura e DAP.
\end{abstract}

Palavras-chave: Açaí; Sensoriamento remoto; Distribuição espacial; Amazônia

\begin{abstract}
Euterpe precatoria Mart., is used in the cosmetic and food industry and has been increasingly exploited in the Amazonian environment. The objective of this work is to analyze the spatial-temporal dynamics of the landscape and the effect of fragmentation on the structure and pattern of spatial distribution of Euterpe precatoria, using a forest fragment in the municipality of Alta Floresta, MT state. Orbital images of Landsat-2/MSS satellite from 1975, Landsat-3/MSS from 1979, Landsat-5/TM from 1985, 1990, 1995, 2000, 2005 and 2010 and from Landsat-8/OLI from 2017 were used. From the data generated by means of remote sensing, the Anthropic Transformation Index -ITA and Linear Regression were calculated. Two plots of $200 \mathrm{~m}$ x $25 \mathrm{~m}$ were drawn in the edge-inland direction. The horizontal and vertical structure of the

Licenciada em Geografia, MSc., Professora da Rede Estadual de Ensino/SEDUC, Doutoranda em Biodiversidade e Biotecnologia da Amazônia Legal - PPG-BIONORTE, Rua Rio Jordão, s/n, bairro Bom Pastor, CEP 78580-000, Alta Floresta (MT), Brasil. jackcochev@gmail.com (ORCID: 0000-0003-0223-4997)

II Bióloga, Dra., Professora do Programa de Pós-Graduação PPG-BIONORTE, Universidade do Estado de Mato Grosso, Rod. MT 208, KM 143, s/n, Jardim Tropical, CEP 78580-000, Alta Floresta (MT), Brasil. anabanrossi@unemat.br (ORCID: 0000-0002-8318-5375)

III Biólogo, Mestrando do Programa de Pós-Graduação em Genética e Melhoramento de Plantas Universidade Estadual do Norte Fluminense Darci Ribeiro, Av. Alberto Lamego, 2000, Parque Califórnia, CEP 28013-600, Campos dos Goytacazes (RJ), Brasil. alexsouzarodrigues@outlook.com (ORCID: 0000-0003-4040-5654)

IV Bióloga, MSc., Doutoranda em Biodiversidade e Biotecnologia da Amazônia Legal - PPG-BIONORTE; Universidade do Estado de Mato Grosso, Rod. MT 208, KM 143, s/n, Jardim Tropical, CEP 78580-000, Alta Floresta (MT), Brasil. kellimuller@hotmail.com (ORCID: 0000-0003-0545-6130) Geógrafa, Drª.., Professora do Programa de Pós-Graduação em Geografia, Universidade do Estado de Mato Grosso, Av. Santos Dumont, s/n, bairro Santos Dumont, CEP 78200-000, Cáceres (MT), Brasil. ssneves@unemat.br (ORCID: 0000-0002-2065-244X)
\end{abstract}


species was characterized by means of the analyzes: number of individuals, density, basal area, diameter distribution and spatial distribution. The spatial distribution pattern was estimated by the Morisita index, with a significance level of 5\%. The first anthropic activities around the study fragment began in 1984 with the opening of the MT-325 highway. The intensification of land use contributed to the reduction of vegetation, which most critical period occurred between 1990 and 2000. We sampled 102 individuals in plot A and 218 in plot B. The density was higher in plot B. The two plots had a larger number of individuals distributed in the first diametric class. The analyzed population presented two spatial distribution patterns, random pattern in plot A and aggregate pattern in the B. Euterpe precatoria plot is balanced on the process of succession and the recruitment of individuals, since the values of the 'q' ratio show that there are a large number of individuals in the first height classes and DAP.

Keywords: Açaí; Remote sensing; Spatial distribution; Amazon

\section{Introdução}

A fragmentação na Amazônia brasileira tornou-se nas últimas décadas objeto de vários estudos, pois é a maior floresta tropical do mundo e agrega a maior diversidade biológica mundial. De acordo com os dados do Monitoramento da Amazônia Brasileira por Satélite - PRODES, desenvolvido pelo Instituto Nacional de Pesquisas Espaciais - INPE e Ministério do Meio Ambiente - MMA, no período de 1988 a 2017, Mato Grosso é o segundo estado que apresenta maior taxa de desmatamento em todo o território da Amazônia Legal, cujas maiores taxas de desmatamento foram de $10,405 \mathrm{~km}^{2}$ no ano de 2003 e $11,814 \mathrm{Km}^{2}$ em 2004 . O processo de ocupação na Amazônia foi intensificado a partir da década de 1980 e permanece nos dias atuais, situação que tem contribuído na fragmentação florestal e interferido na conservação e preservação de espécies endêmicas que ocorrem somente nos ambientes amazônicos (LAURANCE et al., 2011).

Os efeitos da fragmentação florestal na matriz da paisagem causam isolamento de comunidades biológicas, redução na biodiversidade da fauna e flora, alterações nos processos biológicos e interferência na estrutura populacional vegetal, proporcionando o desenvolvimento de novos padrões de distribuição e estrutura de espécies vegetais em diferentes ambientes (CARVALHO; NASCIMENTO, 2009).

Dentre os métodos de análise da fragmentação florestal, as tecnologias espaciais são importantes ferramentas, com destaque para as imagens de sensoriamento remoto, que possibilitam a observação em tempo real de remanescentes florestais, análise espaço-temporal das transformações da paisagem, avaliação dos padrões de uso e cobertura da terra, quantificação da estrutura e padrões de distribuição da paisagem (PIROVANI et al., 2012). Na execução dos tipos de análises citadas é indispensável a utilização do Sistema de Informação Geográfica (SIG), que proporciona a extração de dados das imagens de sensoriamento remoto e a organização destes em Banco de Dados Geográficos (BDG).

Quanto ao efeito da fragmentação florestal sobre comunidades arbóreas, este pode ser observado por meio da análise da estrutura diamétrica, densidade de indivíduos, área basal e distribuição espacial, o que contribui no fornecimento de informações sobre a espécie ou comunidade em estudo, observando os efeitos e prevendo consequências futuras (ABREU, 2014).

$\mathrm{O}$ açaí (Euterpe precatoria Mart.), espécie investigada neste estudo, é hiperdominante da Amazônia (STEEGE et al., 2013), muito utilizado na culinária desde a época da exploração da seringueira, o que motivou o interesse para sua comercialização. Suas partes são utilizadas para o consumo alimentício, a estipe (palmito) e fruto (polpa); extração madeireira (ARRUDA; SILVA; SANDER, 2014) e suas raízes são de uso medicinal (LORENZI, 2010). No município Mato-Grossense de Alta Floresta Euterpe precatoria ocorre de forma natural em áreas de terra firme e em grande abundância, sendo pouco explorado comercialmente pela população local, diferentemente de outras localidades amazônicas em que apresenta alto valor comercial e vem sendo cada vez mais explorado (AZEVÊDO et al., 2017). A sua distribuição geográfica ocorre nos países: Colômbia, Bolívia e no Brasil, abrangendo os estados Acre, Amazonas, Rondônia, Mato Grosso, Pará e Maranhão. 
Diante do exposto, o objetivo deste trabalho é analisar a dinâmica espaço-temporal da paisagem e o efeito da fragmentação na estrutura e no padrão de distribuição espacial da espécie Euterpe precatoria, tendo como modelo um fragmento florestal no município de Alta Floresta-MT.

\section{Material e métodos}

\section{Área de Estudo}

O município mato-grossense de Alta Floresta possui área territorial de $9.212,45 \mathrm{Km}^{2}$, com 42.718 habitantes urbanos e 6.446 na área rural (IBGE, 2017). É a cidade-polo da região de planejamento II- Norte do estado de Mato Grosso (MATO GROSSO, 2017), sendo formada por 15 municípios (Figura 1).

\section{Figura 1 - Localização geográfica da área de estudo.}

Figure 1 - Geographical location of the study area.

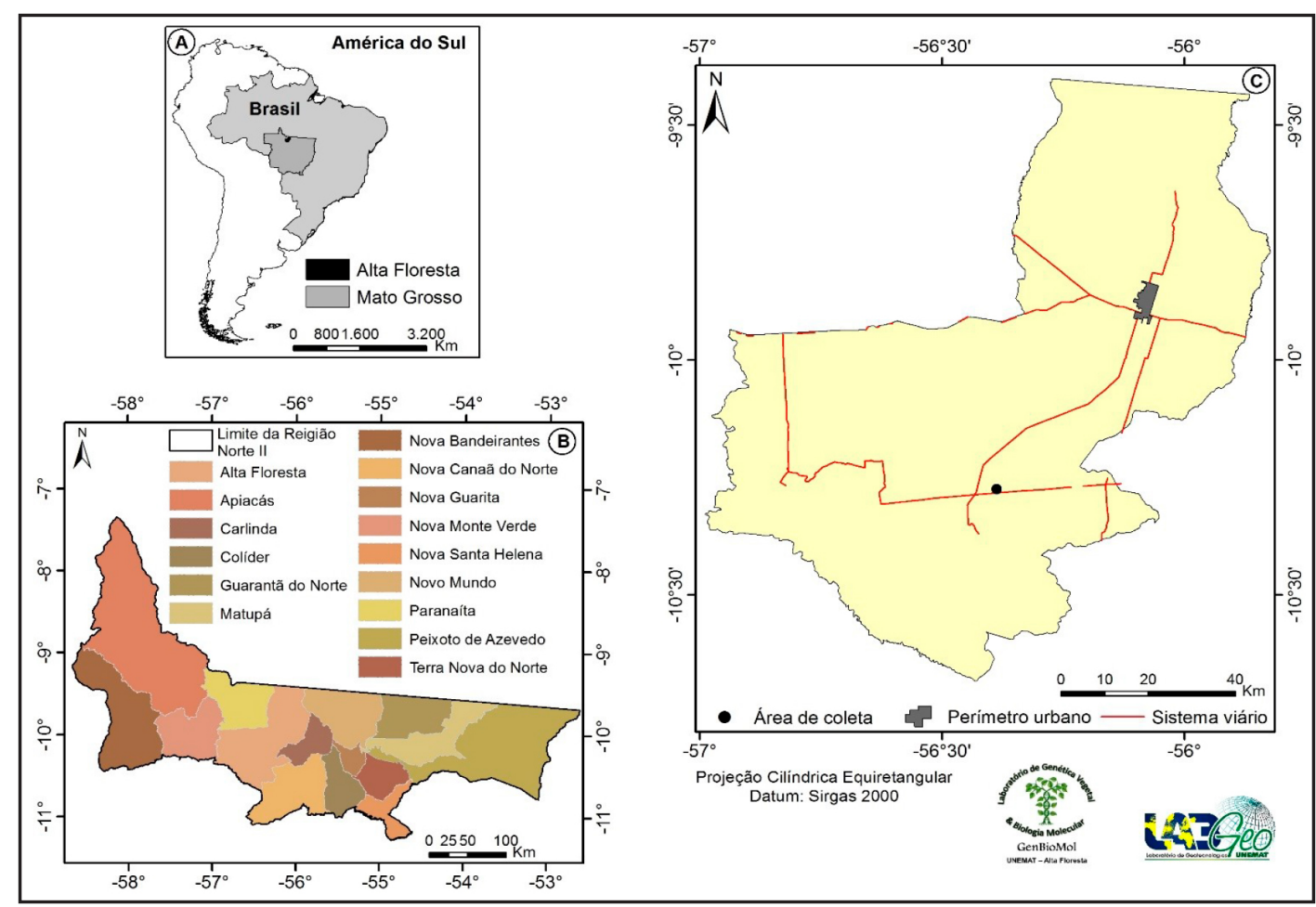

Fonte: IBGE (2017)

A) O município de Alta Floresta no contexto de Mato Grosso e da América do Sul; B) Região Norte de planejamento e seus municípios constituintes; C) Município de Alta Floresta com destaque para cidade e área de estudo

A). The municipality of Alta Floresta, Mato Grosso state,South America; B) North Planning Region and its constituent municipalities; C) Municipality of Alta Floresta detach the city and study area.

A temperatura média anual de Alta Floresta varia entre $19,6^{\circ} \mathrm{C}$ a mínima e $32,4^{\circ} \mathrm{C}$ a máxima, com precipitações no período de maio a setembro, que variam entre 250 a $300 \mathrm{~mm}$, e nos meses de outubro a abril, de 900 a $1000 \mathrm{~mm}$, com a média anual de $2200 \mathrm{~mm}$ (TARIFA, 2011). A vegetação é formada por Florestas Ombrófilas Abertas e Densas, Florestas Estacionais e Formações Secundárias (BRASIL, 2007). São solos predominantes são os: Podzólico Amarelo e 
Vermelho Amarelo, Latossolo e Hidromórficos (NOVAES FILHO et al., 2007). A geomorfologia é formada pelas seguintes formações: Planalto Apiacás-Sucurundi e a Depressão Interplanáltica Amazônia Meridional (ROSS, 2005). A hidrografia é constituída pelos tributários do rio Teles Pires e seus afluentes, quais sejam: Paranaíta, Apiacás, Carlinda, Cristalino e Santa Helena (FERREIRA, 2001).

O fragmento florestal utilizado como modelo neste estudo, dista $60 \mathrm{~km}$ do perímetro urbano de Alta Floresta. Totaliza uma área de 2.376,03 hectares e o seu entorno é utilizado para pastagem e atividade garimpeira. Está situado próximo à comunidade rural denominada Pista do Cabeça (AFPC), onde são desenvolvidas atividades extrativistas e agropecuárias.

\section{Dinâmica espaço-temporal da paisagem no fragmento de estudo}

Para o estudo da dinâmica espaço-temporal da paisagem no fragmento florestal utilizaram-se imagens de sensoriamento remoto do satélite Landsat-2/MSS do ano de 1975 e Landsat-3/MSS do ano de 1979 com resolução espacial de 80 m; do Landsat-5/TM dos anos de 1985, 1990, 1995, 2000, 2005 e 2010, e do Landsat-8/OLI do ano de 2017, com resolução espacial de $30 \mathrm{~m}$. As imagens foram utilizadas para mapeamento e classificação da cobertura vegetal e uso da terra no entorno do fragmento. As imagens foram adquiridas gratuitamente no site do Instituto Nacional de Pesquisas Espaciais - INPE (http://www.dgi.inpe.br).

Para definição dos anos consideraram-se os antecedentes socioeconômicos ocorridos nos períodos, de acordo com a proposta de Cochev et al. (2014):

- 1976 a 1984: fundação do município, quando houve intensa transformação derivada da implantação de áreas agricultáveis;

- 1985 a 1994: extração aurífera e vegetal (madeira e agricultura) no município e no entorno, havendo migração da população para as áreas de garimpo;

- 1994 a 2000: intensificação da extração madeireira;

- a partir de 2000: alteração da dinâmica produtiva agrícola estadual, implicando na constituição de propriedades de uso intensivo e extensivo da terra e inserção de novas políticas ambientais.

As imagens orbitais foram georreferenciadas utilizando como base a imagem de 2015 do Landsat/8-OLI, recortadas, segmentadas e classificadas no software Spring 5.3 do INPE. Na segmentação foram utilizados os parâmetros de similaridade 10 e área 10, aplicando o método crescimento de regiões (interpolador Bhattacharya). A determinação e o recorte do tamanho da área estudo foram realizados a partir do mapeamento do fragmento em que foi realizada a coleta de dados dos espécimes de Euterpe precatoria e a partir da delimitação de um buffer de $1000 \mathrm{~m}$ no entorno do fragmento, considerando o raio de deslocamento dos dispersores e polinizadores da espécie em estudo.

$\mathrm{Na}$ classificação das imagens dos anos selecionados para estudo, as classes temáticas foram agregadas em três categorias: uso agropecuário, que engloba as atividades agrícolas (pastagens e agricultura), vegetação (florestas); e corpos d'água (córregos, lagoas e nascentes). Os arquivos vetoriais gerados foram exportados no formato shapefile (.shp) e no ArcGis, versão 10.4 (ENVIRONMENTAL SYSTEMS RESEARCH INSTITUTE, 2016), foram efetuados os processos de edição, quantificação e geração dos mapas. Os cálculos das estatísticas descritivas e avaliação do Índice de Transformação Antrópica foram realizados por meio dos arquivos dbf, que compõe o shapefile das categorias temáticas.

Os cálculos do Índice de Transformação Antrópica - ITA (LÉMECHEV, 1982), modificado por Mateo (1991), foram aferidos a partir dos valores de área em percentagem (\%) de cada categoria temática através da fórmula (1):

$$
I T A=\frac{\sum(\% \text { Uso } * \text { Peso })}{100}
$$

em que: Uso = área em valores percentuais de cada classe; Peso = valores atribuídos aos diferentes tipos de cobertura vegetal e uso da terra, variando de 1 a 10 , em que 10 indica as maiores pressões. 
Foram atribuídos os seguintes valores para as classes: corpos d'água (2); vegetação (1) e uso agropecuário (6,7), de acordo com os parâmetros desenvolvidos por Gouveia, Galvanin e Neves (2013). A transposição dos índices para valores qualitativos foi realizada a partir do método quartis, realizado por Cruz et al. (1998) sendo: pouco degradada $(0 \vdash 2,5)$, regular $(2,5 \vdash$ 5), degradada $(5 \vdash 7,5)$ e muito degradada $(7,5 \vdash 10)$.

Aplicou-se nas categorias temáticas a análise de Regressão Linear para geração das projeções futuras relativas ao estado de antropização da paisagem na área de estudo, considerando intervalos de 5 anos, até 2050. De acordo com La Peternelli e Mello (2007), consiste em uma análise estatística com o objetivo de relacionar uma variável dependente com uma ou mais variáveis independentes. Neste estudo, a variável dependente são os valores obtidos via ITA e as independentes os períodos das imagens de sensoriamento remoto investigados. Como validação do modelo proposto foi aplicado o coeficiente de determinação $\mathrm{R}^{2}$, sendo que o valor dos resultados quanto mais próximo de 1 indica que o modelo proposto é adequado para descrever o fenômeno (LA PETERNELLI; MELLO, 2007).

\section{Amostragem para Análise da Estrutura Populacional e Distribuição Espacial de Euterpe precatoria}

Foram demarcadas e georreferenciadas duas parcelas de $200 \mathrm{~m}$ de comprimento por $25 \mathrm{~m}$ de largura, no sentido borda-interior. O Sistema de Posicionamento Global-GPS foi configurado com Datum Sirgas 2000 e sistema de coordenadas geográficas. Após a demarcação da primeira parcela (200 m X $25 \mathrm{~m}$ ) na borda do fragmento (parcela A), foi medido, no sentido interior do fragmento, $50 \mathrm{~m}$ de distância para a alocação da segunda parcela (parcela B). Cada parcela possuía uma área de 0,5 hectare, totalizando 1,0 hectare. A escolha pelo método de parcelas permite um mapeamento mais rápido e eficiente das árvores no campo, mostrando-se satisfatório ao avaliar a comunidade arbórea (RODRIGUES, 1991) em estudo.

Foram amostrados todos os indivíduos de Euterpe precatoria com a circunferência a altura do peito $(\mathrm{CAP}) \geq 0,5 \mathrm{~cm}$ que ocorreram nas parcelas, mensurados com fita métrica com precisão milimétrica, e registradas as alturas, que foram estimadas por um único pesquisador, durante todas as campanhas de campo.

Todos os indivíduos amostrados foram marcados com uma etiqueta de plástico, possuindo um número identificador, a fim de possibilitar futuros estudos de dinâmica da comunidade florestal. Foi realizada ainda a coleta de material botânico fértil e depositado no Herbário da Amazônia Meridional (HERBAM) da Universidade do Estado de Mato Grosso, Campus de Alta Floresta, para a confirmação da identificação botânica por meio de comparações com o acervo do HERBAM, e por consultas à literatura e especialistas.

\section{Determinação da estrutura horizontal e vertical de Euterpe precatoria}

A estrutura horizontal e vertical de Euterpe precatoria caracterizou-se por meio das análises dos seguintes parâmetros: NI (Número de Indivíduos), D (Densidade); G (Área basal), Distribuição Diamétrica (DD) e Distribuição Espacial (DE).

O estudo da distribuição diamétrica no ambiente foi obtido através da fórmula de Spiegel:

$$
I C=\frac{A}{n c}
$$

em que: $\mathrm{A}$ = amplitude, $\mathrm{nc}=$ número de classes.

O número de classes a serem utilizadas foi calculado de acordo com a metodologia 
descrita por Higuchi et al. (2008), que consideram: $\mathrm{n}$ classes $=1+3,33 \log \mathrm{N}$ ( $\mathrm{N}=$ número de indivíduos), sendo o intervalo entre as classes ajustado segundo o número de classes. A análise da distribuição diamétrica foi realizada por meio de histogramas, com número de indivíduos por centro de classes de diâmetro, iniciando pelo diâmetro mínimo de inclusão para a espécie.

Após a obtenção da frequência absoluta e relativa, obteve-se o quociente 'q' de D’Liocourt (DE LIOCOURT, 1898; MEYER, 1952), que consiste na divisão do número de indivíduos de uma classe pelo número de indivíduos da classe anterior, através da fórmula:

\section{$\mathrm{Ni}$ \\ $\mathrm{N} i+1$}

em que: $\mathrm{Ni}$ = número de árvores da iésima classe de diâmetro e $\mathrm{Ni}+1$ = número de árvores da iésima classe mais um de diâmetro subsequente

Segundo Felfili, Silva Junior e Nogueira (1998), considera-se balanceada uma população na qual a redução de indivíduos mantém-se em uma constante de uma classe para outra e, uma situação não balanceada, a redução seria proporcional ao aumento de diâmetro.

O padrão de distribuição espacial foi estimado por meio do índice de Morisita (Id), conforme recomendações de Brower e Zar (1977):

$$
I d=\frac{n\left(\sum_{i=\chi^{2}}^{\mathcal{S}}-\mathcal{N}\right)}{\mathcal{N} *(\mathcal{N}-1)}
$$

em que: $\mathrm{Id}=$ índice de Morisita; $\mathrm{n}$ = número total de parcelas amostradas; $\mathrm{N}=$ número total de indivíduos, contidos em $\mathrm{n}$ parcelas; $\mathrm{X}^{2}$ = quadrado do número dos indivíduos por parcela; $\mathrm{s}=$ número de indivíduos amostrados.

A significância dos valores calculados para o índice de Morisita (Id) foi obtido mediante o teste do qui-quadrado, em um nível de significância de 0,05 de probabilidade de erro. A interpretação do valor do qui-quadrado baseou-se nos seguintes parâmetros: se o valor calculado for menor que o valor tabelado, o (Id) não difere significativamente de 1 e a espécie apresenta um padrão de distribuição aleatório. Porém, se o valor do qui-quadrado for maior que o valor tabelado, a espécie possui um padrão de distribuição agregada, a distribuição é uniforme quando Id = 1 (BROWER; ZAR, 1977).

\section{Resultados e discussão}

\section{Análise espaço-temporal da paisagem no fragmento florestal com ocorrência de Euterpe precatoria}

Ao longo dos anos de estudo, a paisagem de entorno e no fragmento florestal foi transformada, em que extensões recobertas com vegetação natural foram convertidas em áreas com usos antrópicos.

Nos primeiros anos após a emancipação de Alta Floresta (1975 a 1979), a área municipal apresentou os primeiros impactos da ação antrópica em alguns pontos, como: a consolidação do espaço urbano e as atividades agropecuárias (COCHEV et al., 2014). Contudo, a área deste estudo apresentava-se com todo seu entorno vegetado nesse período (Figura 2). As primeiras atividades antrópicas no entorno do fragmento iniciaram-se a partir de 1984 com a abertura da rodovia MT-325, cujo percentual de uso agropecuário foi de $1,73 \%$. 
Figura 2 - Cobertura vegetal e uso temporal da terra do entorno e no fragmento modelo, com ocorrência de Euterpe precatoria, no período de 1975-2015.

Figure 2 - Vegetative cover and temporal use of the surrounding land and the model fragment, with Euterpe precatoria, in the period 1975-2015.

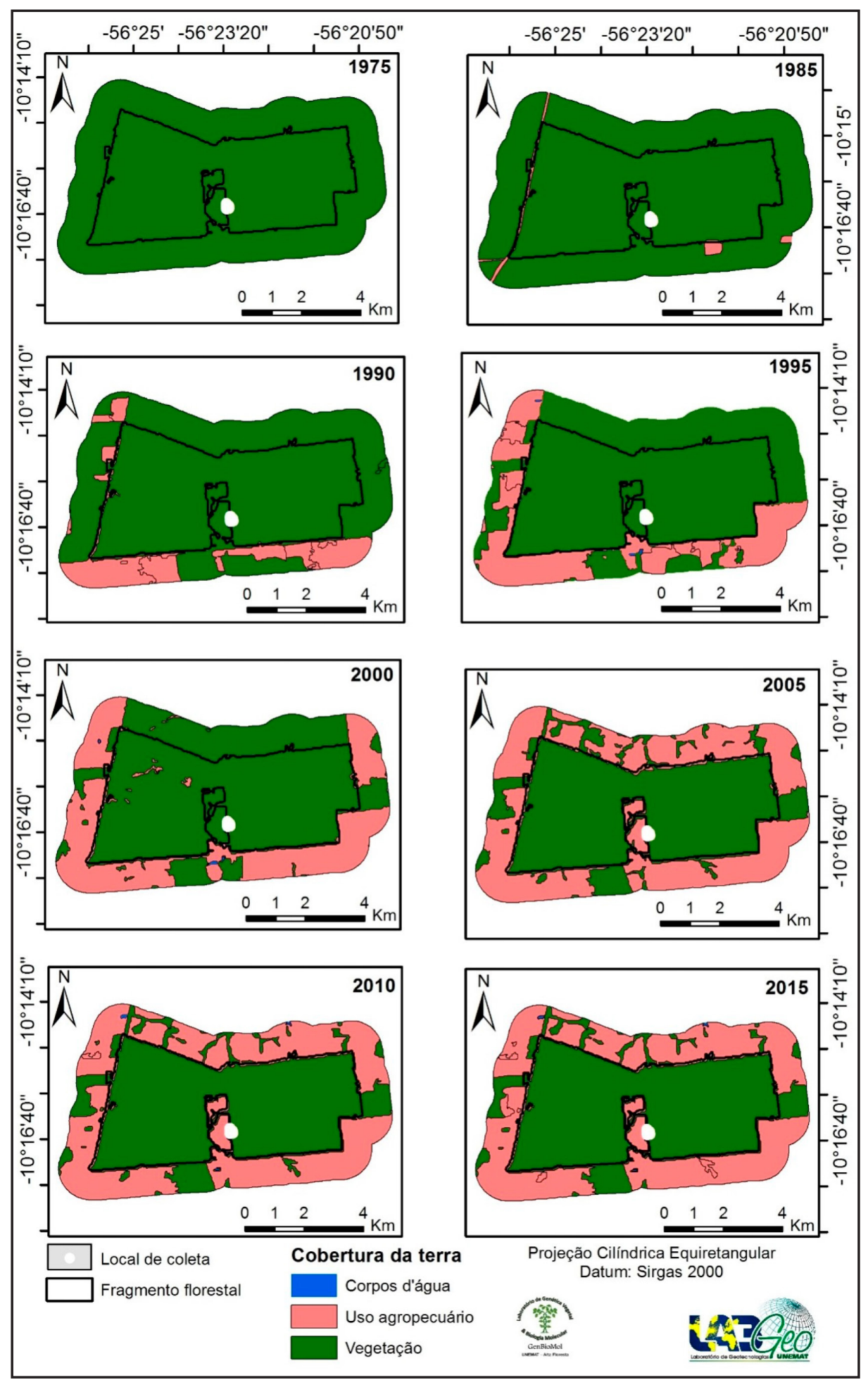

Fonte: Autores (2019) 
Em seu estudo sobre o desmatamento na Amazônia brasileira, Thery (1997) apontou a correlação entre o desmatamento e a abertura de estradas, sendo que a abertura das estradas possibilitava o tráfego facilitando primeiramente o transporte de madeira e em seguida o escoamento de produtos do agronegócio. O mesmo foi verificado por Freitas, Hawbaker e Metzger (2010) em um estudo realizado sobre o efeito da fragmentação na Mata Atlântica, em que as estradas apresentam efeitos em longo prazo e servem de atrativo para as mudanças na dinâmica da paisagem, por apresentar diversos padrões de distribuição ao longo do tempo e no espaço, de forma a atender à demanda política, econômica e social. Nessa perspectiva, nas Figuras 2 e 3 , em que está representada a dinâmica da paisagem de entorno e do fragmento florestal em estudo, observa-se que a fragmentação ocorreu ao longo da estrada e, isso se deve às atividades econômicas desenvolvidas, tais como pecuária e garimpo.

No decorrer dos períodos analisados ocorreu um aumento de aproximadamente $10 \%$ na classe uso agropecuário, com maior intensificação das atividades agropecuárias, no período de 1995 a 2010 (Tabela 1). A intensificação do uso da terra contribuiu para a redução da vegetação e o aparecimento de pequenos corpos d'água, em que o período analisado mais crítico ocorreu entre 1990 e 2000 (Tabela 2).

\section{Tabela 1 - Resultados em percentual (\%) da análise de cobertura vegetal e uso da terra no} fragmento de estudo com ocorrência de Euterpe precatoria.

Table 1 - Results in percentage (\%) of the vegetation cover and land use analysis in the study fragment with occurrence of Euterpe precatoria.

\begin{tabular}{lcccccccccc}
\hline \multicolumn{1}{c}{ Classes (\%) } & $\mathbf{1 9 7 5}$ & $\mathbf{1 9 7 9}$ & $\mathbf{1 9 8 4}$ & $\mathbf{1 9 9 0}$ & $\mathbf{1 9 9 5}$ & $\mathbf{2 0 0 0}$ & $\mathbf{2 0 0 5}$ & $\mathbf{2 0 1 0}$ & $\mathbf{2 0 1 5}$ & $\mathbf{2 0 1 6}$ \\
\hline $\begin{array}{l}\text { Uso } \\
\text { agropecuário }\end{array}$ & 0 & 0 & 1,73 & 13,41 & 23,48 & 29,73 & 42,15 & 42,85 & 43,60 & 46,55 \\
Vegetação & 100 & 100 & 98,27 & 86,59 & 76,43 & 70,19 & 57,85 & 57,07 & 56,32 & 53,32 \\
Água & 0 & 0 & 0 & 0 & 0,07 & 0,08 & 0,08 & 0,08 & 0,08 & 0,08 \\
\hline
\end{tabular}

O aparecimento de corpos d'água a partir de 1995 (Figuras 2 e 3) decorreu da retirada da vegetação do entorno de nascentes e/ou de pequenos canais hídricos, contudo, em todos os anos analisados, esta foi a classe que apresentou os menores Índices de Transformação Antrópica (Tabela 2), devido às leis de proteção das Áreas de Preservação Permanente (APPs), que permitiram que estas áreas fossem mais conservadas.

A abertura da rodovia MT-325 proporcionou aumento do ITA para todas as classes analisadas (Tabela 2), apresentando-se a paisagem de pouco degradada a regular no período analisado. Para a classe uso agropecuário, o aumento do ITA ocorreu consideravelmente no período de 2005 a 2015, cujo uso da terra para produção agropecuária aumentou para a consolidação do mercado, sendo reflexo da intensa atividade de extração mineral e vegetal nos anos anteriores, e a intensificação das atividades agropecuárias a partir de 2000 (COCHEV et al., 2014). Devido à expansão do uso agropecuário desenvolvido no entorno do fragmento florestal, o ITA passou de pouco degradado em 1995 a regular em 2005, indicando redução da área recoberta por cobertura vegetal natural. Ao analisar composição da paisagem nos anos de 2010 e 2015 na Figura 2 e o ITA na (Tabela 2), observa-se que ocorreram alterações na paisagem relativa à supressão da vegetação natural e introdução do uso agropecuário. 


\section{Tabela 2 - Índice de Transformação Antrópica (ITA) no fragmento florestal de estudo com ocorrência de Euterpe precatoria no município de Alta Floresta-MT.}

Table 2 - Anthropogenic Transformation Index (ITA) in the study forest fragment with occurrence of Euterpe precatoria in the municipality of Alta Floresta, MT state.

\begin{tabular}{|c|c|c|c|c|c|c|c|c|c|}
\hline \multirow{2}{*}{ Classes temáticas } & \multicolumn{9}{|c|}{ ITA } \\
\hline & 1975 & 1979 & 1984 & 1990 & 1995 & 2000 & 2005 & 2010 & 2015 \\
\hline Uso agropecuário & 0 & 0 & 0,12 & 0,9 & 1,57 & 1,99 & 2,82 & 2,87 & 2,92 \\
\hline Vegetação & 1 & 1 & 0,98 & 0,87 & 0,76 & 0,70 & 0,58 & 0,57 & 0,56 \\
\hline Água & 0 & 0 & 0 & 0 & 0 & 0 & 0 & 0 & 0 \\
\hline Total & 1 & 1 & 1,1 & 1,76 & 2,34 & 2,7 & 3,4 & 3,44 & 3,49 \\
\hline
\end{tabular}

Em geral, o ITA passou de pouco degradado para regular no período analisado (Tabela 2). No período de 1975 a 2000 ocorreu intensificação da retirada da vegetação nativa, corroborando os resultados mostrados na Tabela 1, em que a conversão da vegetação proporcionou o aparecimento de pequenos corpos d'água e a substituição da vegetação nativa em atividades agropecuárias, o que acarretou na interferência do padrão de distribuição espacial dos espécimes de Euterpe precatoria, na densidade dos indivíduos, efeito de borda e perda de diversidade biológica. A conversão da vegetação nativa em áreas produtivas acarreta também em interferências no microclima local, em que as espécies de interesse comercial podem sofrer perdas de indivíduos ou substituição total da espécie ao longo do tempo, que suporte as condições ambientais atuais (LIMA-RIBEIRO, 2008).

A regressão linear (Figura 3) corroborou os resultados apresentados pelo ITA de que ao longo dos anos os usos agropecuários desenvolvidos contribuíram para mudanças no estado de conservação da paisagem, até a década de 2000 e, nos anos de 2005, 2010 e 2015 houve estabilização. O coeficiente de determinação $\left(R^{2}\right)$ foi de 0,9529 (Figura 3) e evidenciou correlação entre o ITA e a ocorrência de Euterpe precatoria no município de Alta Floresta, considerando que há maior quantidade de indivíduos de Euterpe precatoria em áreas que estão sob efeito de borda, que estão situadas no entorno dos fragmentos.

Figura 3 - Regressão Linear das projeções do Índice de Transformação Antrópica no fragmento florestal com ocorrência de Euterpe precatoria, no município de Alta Floresta-MT.

Figure 3 - Linear regression of the projections of the Anthropic Transformation Index on the forest fragment with occurrence of Euterpe precatoria, in the municipality of Alta Floresta, MT state.

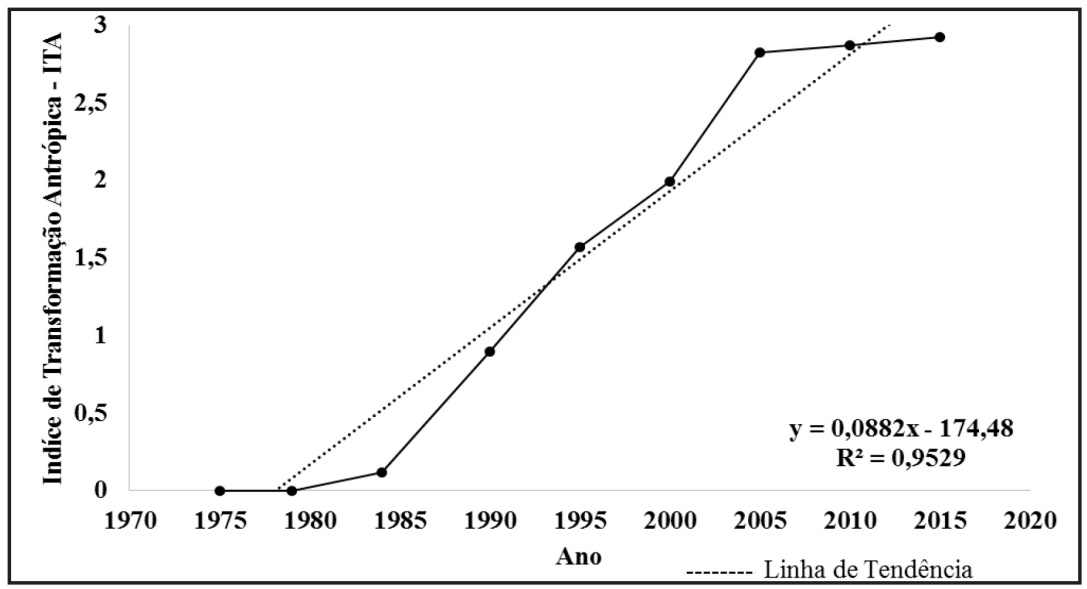

Fonte: Autores (2019) 
A análise da equação de regressão (Tabela 3) dos anos subsequentes a 2015 indicou que até 2030 a paisagem apresentará estado regular de conservação, ou seja, estará estabilizada mesmo havendo ampliação das atividades agropecuárias. Porém, a partir de 2035, haverá mudança de estado para degradado, implicando efetivamente na perda de diversidade biológica de comunidade florestais que ocorrem naturalmente no fragmento de estudo. De acordo com Silva (2013), o aumento do desmatamento no município de Alta Floresta é decorrente do processo de ocupação iniciado a partir de 1970, através da extração aurífera e madeireira e, atualmente pela expansão agropecuária na região, que proporcionará a degradação de áreas verdes nos próximos anos.

Tabela 3 - Projeção do Índice de Transformação Antrópica de 2020 a 2050.

Table 3 - Projection of the Anthropic Transformation Index from 2020 to 2050.

\begin{tabular}{lccc}
\hline Tempo & Equação de regressão & Projeção do ITA & Índice do ITA \\
\hline $\mathbf{2 0 2 0}$ & $\mathrm{y}=\left(0,0882^{\star} 2020\right)-174,48$ & 3,68 & Regular \\
$\mathbf{2 0 2 5}$ & $\mathrm{y}=\left(0,0882^{\star} 2025\right)-174,48$ & 4,13 & Regular \\
$\mathbf{2 0 3 0}$ & $\mathrm{y}=\left(0,0882^{\star} 2030\right)-174,48$ & 4,57 & Regular \\
$\mathbf{2 0 3 5}$ & $\mathrm{y}=\left(0,0882^{\star} 2035\right)-174,48$ & 5,01 & Degradada \\
$\mathbf{2 0 4 0}$ & $\mathrm{y}=\left(0,0882^{\star} 2040\right)-174,48$ & 5,45 & Degradada \\
$\mathbf{2 0 4 5}$ & $\mathrm{y}=\left(0,0882^{\star} 2045\right)-174,48$ & 5,89 & Degradada \\
$\mathbf{2 0 5 0}$ & $\mathrm{y}=\left(0,0882^{\star} 2050\right)-174,48$ & 6,33 & Degradada \\
\hline
\end{tabular}

\section{Estrutura populacional de indivíduos de Euterpe precatoria em fragmento florestal no município de Alta Floresta-MT}

A espécie modelo neste estudo, Euterpe precatoria ocorre naturalmente no município de Alta Floresta-MT. No fragmento de estudo, foram amostrados 320 espécimes de Euterpe precatoria em duas parcelas, sendo 102 indivíduos na primeira parcela (A) e 218 na segunda (B). A densidade foi de $204 \pm 5,68$ ind.ha ${ }^{-1}$ na parcela A e $436 \pm 12,15$ ind.ha $^{-1}$ na parcela B. Os valores das estatísticas encontrados para a distribuição da espécie no local de estudo estão apresentados na Tabela 4.

As duas parcelas apresentam um maior número de indivíduos distribuídos na primeira classe diamétrica, sendo mais acentuado na parcela A, que está localizada na borda do fragmento, o que para Nunes et al. (2003) significa que a espécie em estudo encontra-se em estado de sucessão secundária após uma perturbação. Meira et al. (2016) ressaltam ainda, que a maior densidade de indivíduos na primeira classe diamétrica caracteriza que a floresta é detentora de estoque de árvores finas, capazes de substituir árvores de maior porte que venham a ser eliminadas. O fato da espécie em estudo estar em estádio de regeneração, com maior número de indivíduos na primeira classe diamétrica deve-se, provavelmente, ao efeito da fragmentação que ocorre no entorno da área de estudo ou mesmo, aos fatores bióticos (dispersores e polinizadores) e abióticos (radiação solar, pluviosidade, fatores químicos) que atuam sobre a espécie.

Verifica-se que as médias da parcela A foram maiores que da parcela B. Dentre os fatores que podem ter influenciado nas estatísticas analisadas, as condições ambientais sob as quais a espécie encontra-se é fator preponderante, pois segundo Nasi (1993) existem espécies que apresentam caráter de adaptação às variações ecológicas. Outro fator importante a ser observado quanto à estrutura da espécie é a área basal que apresentou a maior média na parcela A. Andrade et al. (2017) no levantamento fitossociológico em área de terra firme com espécies amazônicas 
encontraram para doze espécies da família Arecaceae valores entre $0,03 \mathrm{~cm}^{2} \cdot \mathrm{ha}^{-1}$ a $11,83 \mathrm{~cm}^{2}$. $\mathrm{ha}^{-1}$, sendo que para Euterpe precatoria encontraram o valor de $1,76 \mathrm{~cm}^{2} . \mathrm{ha}^{-1}$, corroborando os valores encontrados neste trabalho. Silva (2013) e Dardengo (2017) para as espécies amazônicas Theobroma speciosum Willd. ex Spreng. e Theobroma subicanum Mart. respectivamente, em três fragmentos florestais urbanos município de Alta Floresta e no Parque Nacional do Juruena, encontraram valores médios semelhantes para espécies florestais, o que pode representar um padrão característicos de árvores amazônicas.

Tabela 4 - Síntese do inventário florestal da espécie Euterpe precatoria Mart. em duas parcelas em um fragmento florestal no município de Alta Floresta-MT.

Table 4 - Synthesis of the forest inventory of Euterpe precatoria Mart. in two plots in a forest fragment in the municipality of Alta Floresta, MT state.

\begin{tabular}{|c|c|c|c|c|c|c|}
\hline Parcelas & Área (ha) & PCD (\%) & Estatísticas & $\operatorname{DAP}(\mathbf{c m})$ & Altura $(\mathbf{m})$ & Área basal $\left(\mathrm{cm}^{2} \cdot \mathbf{h a}^{-1}\right)$ \\
\hline \multirow{5}{*}{ A } & \multirow{5}{*}{0,5} & \multirow{5}{*}{38,24} & Maior & 17,18 & 25 & 232,04 \\
\hline & & & Menor & 1,27 & 1 & 1,27 \\
\hline & & & Média & 4,89 & 5,99 & 28,95 \\
\hline & & & Desvio Padrão & 3,6 & 4,99 & 47,74 \\
\hline & & & $\mathrm{CV} \%$ & 73,61 & 83,4 & 164,89 \\
\hline \multirow{5}{*}{ B } & \multirow{5}{*}{0,5} & \multirow{5}{*}{35,32} & Maior & 17,5 & 30 & 240,72 \\
\hline & & & Menor & 1,59 & 1 & 1,98 \\
\hline & & & Média & 3,85 & 5,6 & 23,75 \\
\hline & & & Desvio Padrão & 2,96 & 4,6 & 36,77 \\
\hline & & & $\mathrm{CV} \%$ & 76,89 & 82,18 & 154,8 \\
\hline
\end{tabular}

Em que: PCD = percentual de indivíduos na primeira classe diamétrica; DAP = diâmetro a altura do peito.

No fragmento vegetal em que foram alocadas as parcelas, a população de Euterpe precatoria apresentou dois padrões de distribuição espacial (Tabela 5), conforme as recomendações de Brower e Zar (1977): na parcela A um padrão aleatório, e na parcela B um padrão agregado. A parcela A está mais próxima da área de pastagem (Figura 4), sendo uma área que sofre com a ação externa das atividades que ocorrem no entorno.

$\mathrm{O}$ padrão da parcela $\mathrm{A}$ e o identificado visualmente em campo corroboram Odum e Barrett (2008), os quais expuseram que a distribuição aleatória permite um afastamento entre os indivíduos e que possuem influência de fatores antrópicos. $\mathrm{Na}$ parcela $\mathrm{B}$, que apresenta padrão agregado, ocorre a tendência em formar grupos e da prole permanecer próxima aos pais (RICKLEFS, 2009).

Pela distribuição espacial, de acordo com a relação DAP x Altura, há maior número de indivíduos distribuídos nas primeiras classes, tanto na parcela A quanto na parcela B (Figura 5). Houve uma correlação entre o DAP x altura dos indivíduos amostrados nas duas parcelas. Nunes et al. (2003) relataram que o excesso de indivíduos nas primeiras classes pode ser indicativo de sucessão secundária após uma perturbação, pois existe uma reserva de estoque de árvores para substituir as que venham a ser eliminadas, seja de forma natural ou em manejo, sendo uma estratégia de perpetuação da espécie. 
Tabela 5 - Distribuição espacial de Euterpe precatoria Mart. em um fragmento florestal no município de Alta Floresta-MT.

Table 5 - Spatial distribution of Euterpe precatoria Mart. in a forest fragment in the municipality of Alta Floresta, MT state.

\begin{tabular}{lcccc}
\hline Parcelas & Índice de Morisita & $\mathbf{X}^{2}$ & $\mathbf{X}$ Tabelado & Distribuição Espacial \\
\hline $\mathbf{A}$ & 1,007 & 3,803 & 7,814 & Aleatório \\
$\mathbf{B}$ & 1,023 & 8,128 & 7,814 & Agregado \\
\hline
\end{tabular}

Fonte: Autores (2019)

Figura 4 - Distribuição espacial das parcelas A e B com os indivíduos de Euterpe precatoria, no município de Alta Floresta-MT.

Figure 4 - Spatial distribution of plots A and B with individuals of Euterpe precatoria, in the municipality of Alta Floresta, MT state.

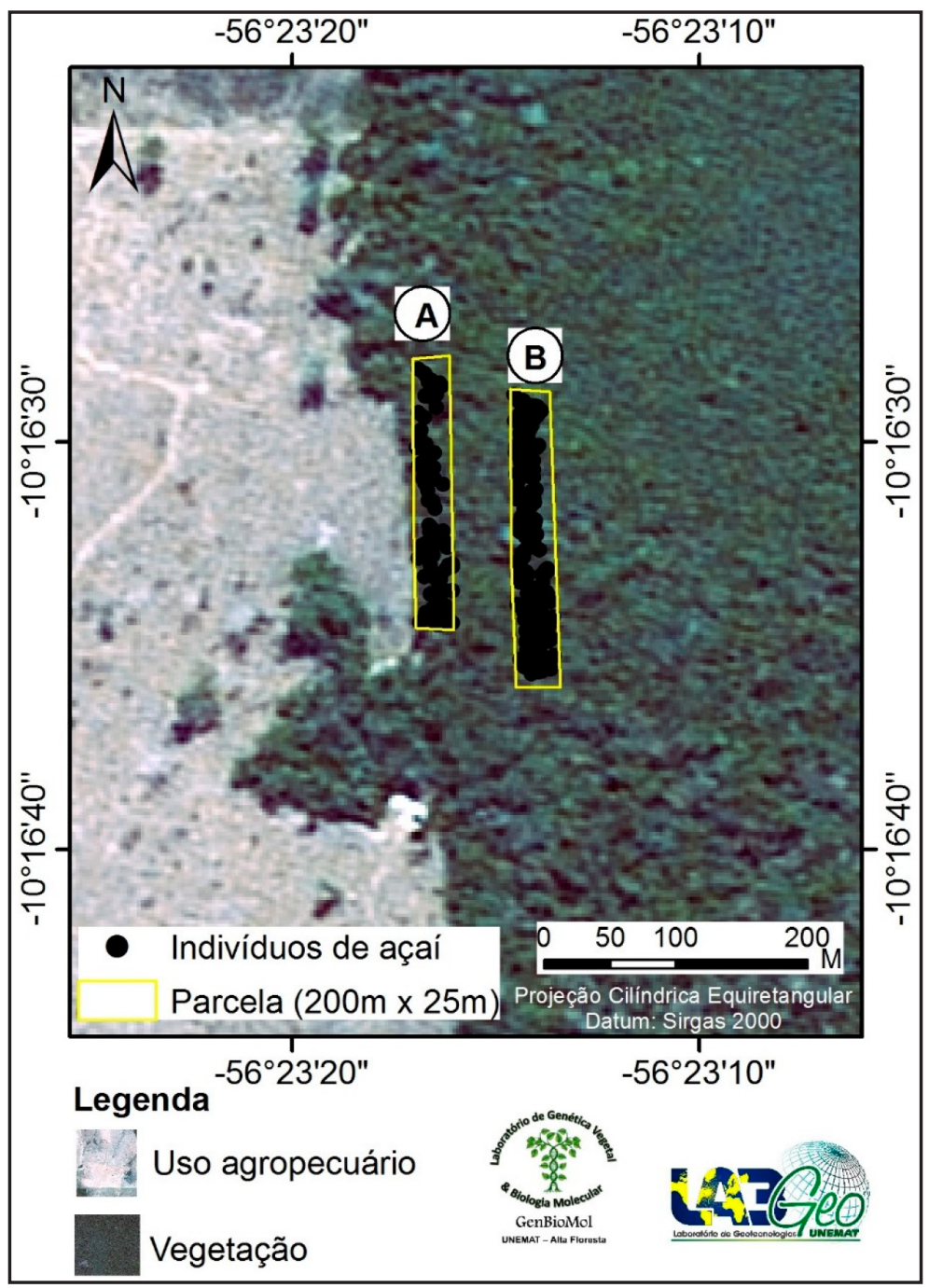

Fonte: Autores (2019) 


\section{Figura 5 - Relação DAP x Altura dos espécimes de Euterpe precatoria.}

Figure 5 - Relationship DAP x Height of Euterpe precatoria specimens.

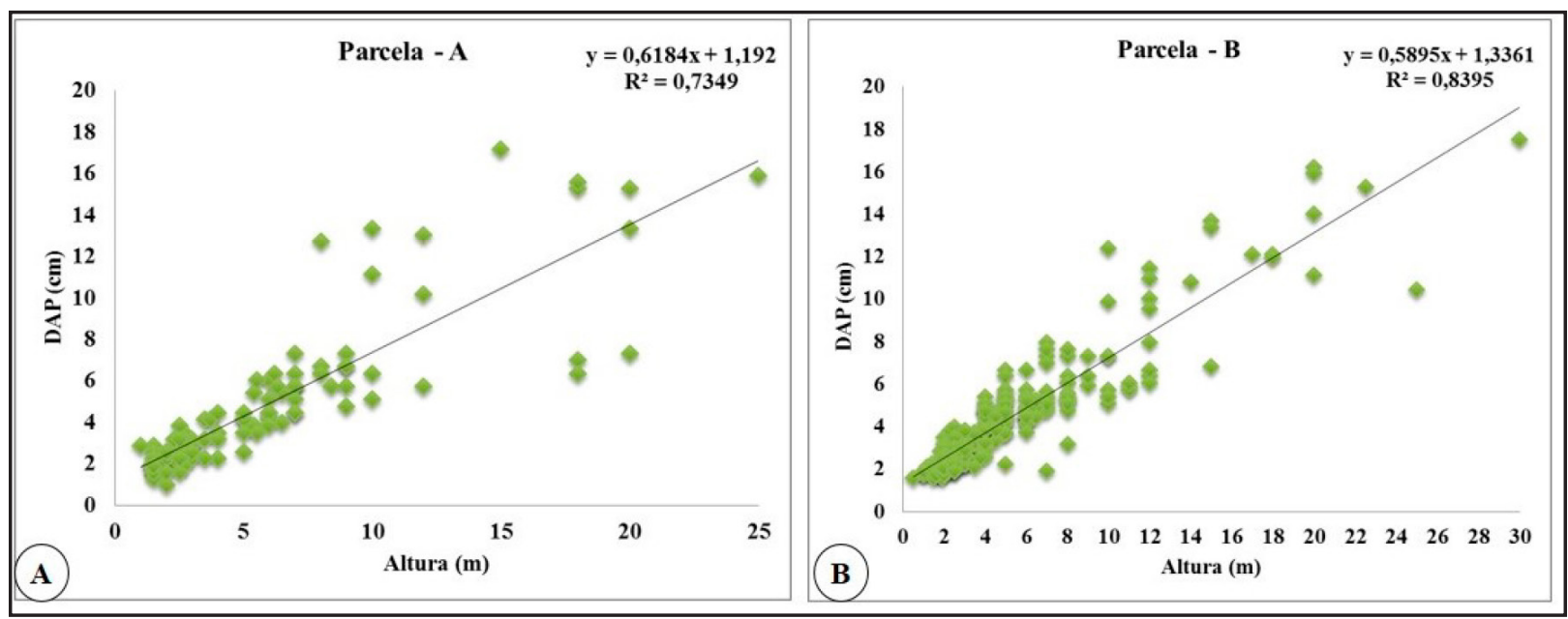

Fonte: Autores (2019)

A: Parcela borda (A); B: Parcela interior (B).

A: Edge plot (A); B: Inside plot (B).

Tanto a distribuição diamétrica (Figura 6) como a altura (Figura 7) dos indivíduos em ambas as parcelas apresentaram o "J-reverso", o que segundo Scolforo (1998), Alves Junior et al. (2009) e Machado et al. (2010) é comum para espécies nativas que não estão em estado de estresse, apresentando uma tendência ao balanceamento entre mortalidade e recrutamento.

Figura 6 - Frequência absoluta e classes de altura dos indivíduos de Euterpe precatoria.

Figure 6 - Absolute frequency and height classes of Euterpe precatoria individuals.

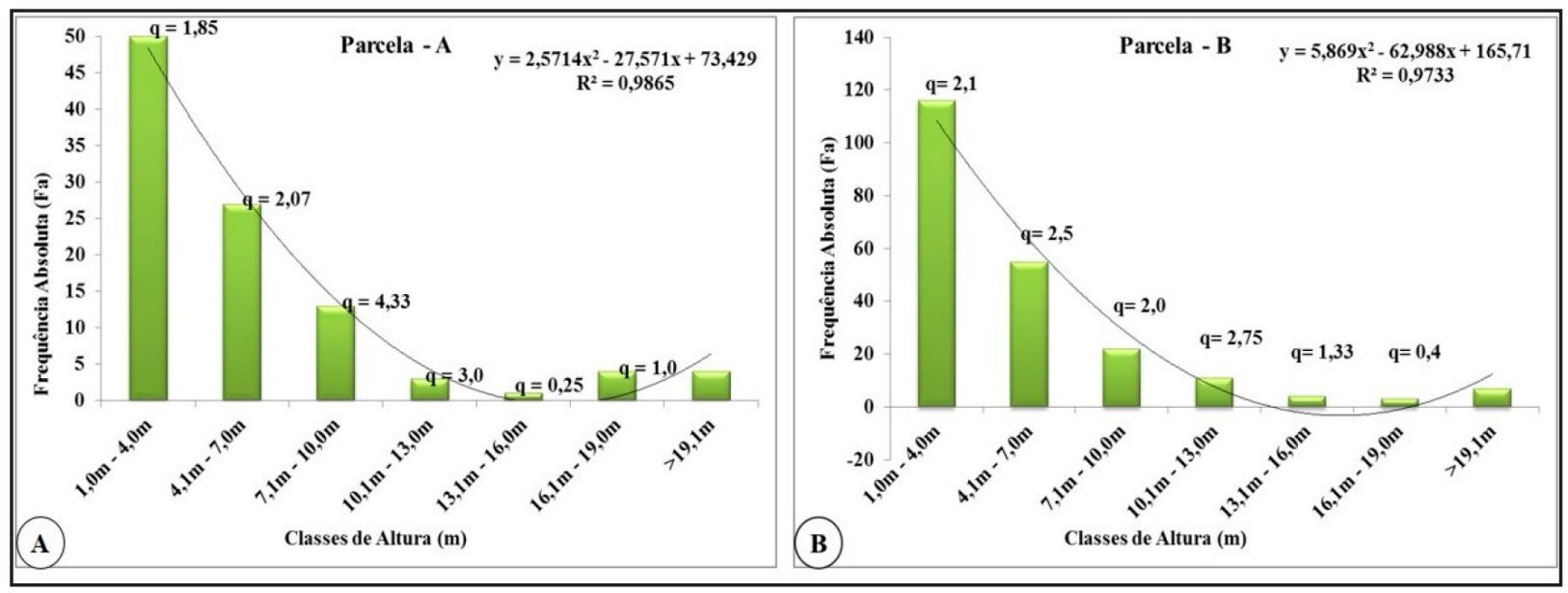

Fonte: Autores (2019)

A: Parcela borda (A); B: Parcela interior (B).

A: Edge plot (A); B: Inside plot (B). 


\section{Figura 7 - Frequência absoluta e classes de DAP dos indivíduos de Euterpe precatoria.}

Figure 7 - Absolute frequency and DAP classes of Euterpe precatoria individuals.

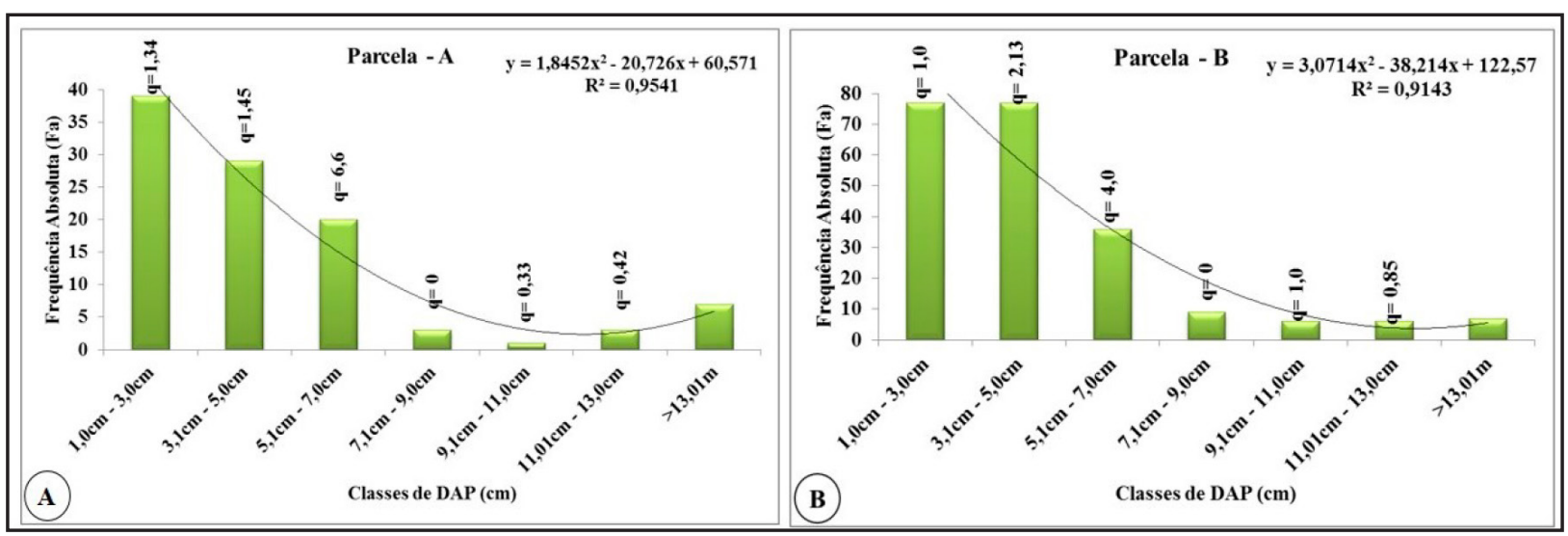

Fonte: Autores (2019)

A: Parcela borda (A); B: Parcela interior (B).

A: Edge plot (A); B: Insideplot (B).

Martins (1991) afirmou que o diâmetro do tronco guarda uma certa proporcionalidade com a idade da árvore, sendo possível afirmar que populações com classes de DAP mais elevadas estão em estádio de sucessão mais avançada. Como as duas populações amostradas apresentaram maior frequência de indivíduos nas classes menores de DAP, pode-se dizer que as duas populações em estudo encontram-se em estádio sucessional jovem, o que pode estar relacionado à estrutura da vegetação e ao estádio de sucessão na área de estudo.

A curva ajustada aos valores de frequência para a parcela A e parcela B das classes diamétricas (Figuras 6 e 7) com a constante 'q' indica que a população apresenta-se balanceada, pois os valores da razão 'q' estão constantes entre as primeiras classes de menores DAP. Contudo, nas classes com maior DAP, verifica-se que há um baixo número de indivíduos por classe, não apresentando a razão 'q' constante, mostrando que há perturbação no ambiente e o que os indivíduos de Euterpe precatoria não estão em equilíbrio, em que quanto maior o valor de 'q' mais severa foi ou está sendo a perturbação.

\section{Conclusões}

No entorno do fragmento florestal, a antropização para fins agropecuários promoveu a supressão da cobertura vegetal, que na atualidade está presente principalmente nas áreas de preservação permanentes situadas ao longo dos cursos hídricos. Caso não haja mudança no desenvolvimento das atividades antrópicas, os cenários futuros apontam para a o aumento do uso agropecuário em detrimento da cobertura vegetal.

A análise da estrutura populacional nas parcelas A e B evidenciou que as mudanças ocorridas no entorno do fragmento têm interferido no padrão de distribuição espacial de Euterpe precatoria.

Euterpe precatoria encontra-se balanceada quanto ao processo de sucessão e recrutamento dos indivíduos, porém, os valores da razão 'q' mostram que há um grande número de indivíduos nas primeiras classes de altura e DAP, apresentando-se balanceadas e nas classes superiores não apresenta recrutamento de indivíduos da classe subsequente, revelando assim que efeitos do ambiente interferem na perpetuação da espécie, principalmente na parcela A. 


\section{Agradecimentos}

A Fundação de Amparo à Pesquisa do Estado de Mato Grosso-FAPEMAT pela concessão de bolsa de doutorado da primeira autora durante o período de outubro de 2015 a março de 2017 e pelo financiamento do projeto de pesquisa: "Dinâmica da paisagem de fragmentos florestais e estrutura genética populacional de duas espécies nativas da Amazônia”, Processo n 229312/2015.

\section{Referências}

ABREU, J. C. et al. Estrutura e distribuição espacial de andirobeiras (Carapa spp.) em floresta de várzea do estuário amazônico. Ciência Florestal, Santa Maria, v. 24, n. 4, p. 1009-1019, out./dez. 2014.

ALVES JUNIOR, F. T. et al. Estrutura diamétrica de um fragmento de Floresta Atlântica em matriz de cana-de-açúcar, Catende-PE. Revista Brasileira de Engenharia Agrícola e Ambiental, Campina Grande, v. 13, n. 3, p. 328-333, 2009.

ANDRADE, R. T. G. et al. Fitossociologia de uma floresta de terra firme na Amazônia SulOcidental, Rondônia, Brasil. Biota Amazônia, Macapá, v. 7, n. 2, p. 36-43, 2017.

ARRUDA, J. C.; SILVA, C. J.; SANDER, N. L. Conhecimento e uso do babaçu (AttaleaSpeciosa Mart.) por quilombolas em Mato Grosso. Fragmentos de Cultura, Goiás, v. 24, n. 2, p. 239-252, 2014.

AZÊVEDO, H. S. F. S. et al. Transferability of heterologous microsatellite loci between species of Euterpe genus. Genetics and Molecular Research, Ribeirão Preto, v. 16, n. 4, p. 1-7, 2017.

BRASIL. Ministério do Meio Ambiente. Secretaria de Políticas para o Desenvolvimento Sustentável. Programa de Zoneamento-Ecológico-Econômico. Caderno temático: biodiversidade no âmbito do zoneamento ecológico-econômico. Brasília: Ministério do Meio Ambiente, 2007. 240 p.

BROWER, J. E.; ZAR, J. H. Field and laboratory methods for general ecology. Iowa: W.C. Brown Company Publishers; Cambridge: Cambridge University Press, 1977. 632 p.

CARVALHO, F. A.; NASCIMENTO, M. T. Estrutura diamétrica da comunidade e das principaispopulações arbóreas de um remanescente de floresta Atlântica Submontana (Silva Jardim-RJ, Brasil). Revista Árvore, Viçosa, MG, v. 33, n. 2, p. 327-337, 2009.

COCHEV, J. S. et al. Sistemas de produção olerícola comercial do município mato-grossense de Alta Floresta, Brasil. Rae’ ga-o espaço geográfico em análise, Curitiba, v. 32, p. 240-266, 2014.

CRUZ, C. B. M. et al. Carga antrópica da bacia hidrográfica da Baía de Guanabara. In: SIMPÓSIO BRASILEIRO DE SENSORIAMENTO REMOTO, 9., 1998, Santos. Anais [...]. Santos: SBSR, 1998. v. 9 , p. 99-109.

DARDENGO, J. D. F. E. et al. Spatial structure of Theobroma subincanum Mart. and Theobroma speciosum Willd. ex Spreng. in the Parque Nacional do Juruena, Mato Grosso state, Brazil. Revista Árvore, Viçosa, MG, v. 41, n. 1, p. 1-10, 2017.

DE LIOCOURT, F. De l'amena gemente dessapinieres. Bulletin Société Franche-Comté et Belfort, [s. 1.], v. 4, p. 396-409, 1898.

ENVIRONMENTAL SYSTEMS RESEARCH INSTITUTE. ArcGis advanced: realease 10.4. Redlands: ESRI, 2016.

FELFILI, J. M.; SILVA JÚNIOR, M. C.; NOGUEIRA, P. E. Levantamento da vegetação arbórea na região de Nova Xavantina, MT. Boletim do Herbário Ezechias Paulo Heringer, Brasília, v. 3, p. 63-81, 1998.

FERREIRA, J. C. V. Mato Grosso e seus municípios. Cuiabá: Buriti, 2001. 660 p. 
FREITAS, S. R.; HAWBAKER, T. J.; METZGER, J. P. Effects of roads, topography, and land use on forest cover dynamics in the Brazilian Atlantic Forest. Forest Ecology and Management, Amsterdam, n. 259, p. 410-417, 2010.

GOUVEIA, R. G. L.; GALVANIN, E. A. S.; NEVES, S. M. A. S. Aplicação do Índice de Transformação Antrópica na análise multitemporal da bacia do córrego do Bezerro Vermelho em Tangará da Serra-MT. Revista Árvore, Viçosa, MG, v. 37, n. 6, 2013.

HIGUCHI, P. et al. Dinâmica da comunidade arbórea em um fragmento de floresta estacional semidecidualmontana em Lavras, Minas Gerais, em diferentes classes de solos. Revista Árvore, Viçosa, MG, v. 32, n. 3, p. 417-426, 2008.

IBGE. IBGE Cidades. Rio de Janeiro, 2017.

LA PETERNELli, L. A.; MELLO, M. P. Conhecendo o R: uma visão estatística. Viçosa, MG: UFV, 2007.

LAURANCE W. F. et al. The fate of Amazonian forest fragments: a 32-year investigation. Biological Conservation, Essex, v. 144, n. 1, p. 56-67, 2011. DOI: http://dx.doi.org/10.1016/j. biocon.2010.09.021.

LEMESHEV, M. Socialismo y Naturaleza. Fundamentos científicos de lautilización socialista de lanaturaleza (en ruso). Moscú: Editorial Misl, 1982. v. 223.

LIMA-RIBEIRO, M. S. Efeitos de borda sobre a vegetação e estruturação populacional em fragmentos de Cerradão no Sudoeste Goiano, Brasil. Revista Acta Botânica Brasilica, Brasília, v. 22 , n. 2 , p. 535-545, 2008.

LORENZI, H. J. Flora brasileira:'Arecaceae' (palmeiras). [S. l.]: Instituto Plantarum, 2010.

MACHADO, E. L. M. et al. Flutuações temporais nos padrões de distribuição diamétrica da comunidade arbóreo-331 arbustivo e de 15 populações em um fragmento florestal. Revista Árvore, Viçosa, MG, v. 34, n. 4, p. 723-732, 2010.

MARTINS, F. R. Estrutura de uma floresta mesófila. Campinas: Unicamp, 1991. 245 p.

MATEO, J. Geoecologia de los paisajes. Mérida: ULA, 1991.

MATO GROSSO. Secretaria de Estado de Planejamento. Regiões de Planejamento de Mato Grosso: 2017. Cuiabá: Secretaria de Estado de Planejamento, 2017. 245 p.

MEIRA, M. R. et al. Caracterização estrutural do BARBATIMÃO (Stryphnodendronadstringens (Mart.) Coville) no cerrado do norte de Minas Gerais. Revista Ciência Florestal, Santa Maria, v. 26, n. 2, p. 627-638, abr./jun. 2016.

MEYER, H. A. Structure, growth and drain in balanced uneven-aged forests. Journal of Forest, [s. 1.], v. 50, p. 85-92, 1952.

NASI, R. Analysis of the spatial structure of a rattan population in a mixed dipterocarp forest of Sabah (Malaysia). Acta Oecologica, Paris, v. 34, n. 1, p. 73-85, 1993.

NOVAES FILHO, J. P. et al. Distribuição de Carbono em Solos sob Floresta Primária na Amazônia Meridional. Revista Árvore, Viçosa, MG, v. 31, n. 1, p. 83-92, 2007.

NUNES, Y. R. F. et al. Variações da fisionomia, diversidade e composição de guildas da comunidade arbórea em um fragmento de floresta semidecidual em Lavras, MG. Acta Botânica Brasílica, Brasília, v. 17, n. 2, p. 213-229, 2003.

ODUM, E. P.; BARRETT, G. W. Fundamentos de Ecologia. São Paulo: Cengage Learning, 2008.

PIROVANI, D. et al. Uso de geotecnologias para estudo da fragmentação florestal com base em princípios de ecologia da paisagem. In: SANTOS, R. A. et al (org.). Geotecnologias aplicadas aos 
recursos florestais. Alegre: CAUFES, 2012. p. 24-41.

RICKLEFS, R. E. A Economia da Natureza. 5. ed. Rio de Janeiro: Guanabara Koogan, 2009. 503 p.

RODRIGUES, R. R. Análise de um remanescente de vegetação natural às margens do rio Passa Cinco, Ipeúna, SP. 1991. Tese (Doutorando em Ciências) - Universidade Estadual de Campinas, Campinas, 1991.

ROSS, J. S. O relevo no processo de produção do espaço. In: MORENO, G.; HIGA, T. C. S. Geografia de Mato Grosso: território, sociedade, ambiente. Cuiabá: Entrelinhas, 2005. p. 218237.

SCOLFORO, S. R. Manejo Florestal. Lavras: UFLA; FAEPE. 1998. 443 p.

SILVA, B. M. et al. Estrutura e padrões de distribuição espacial de duas espécies de Theobroma em um parque de preservação permanente no norte do estado de Mato Grosso. Enciclopédia Biosfera, Goiânia, v. 9, n. 17, p. 2789-95, 2013.

STEEGE, H. et al. Hyperdominance in the AmazonianTree Flora. Science, Cambridge, v. 342, p. 325334, 2013. DOI: 10.1126/science.1243092.

TARIFA, J. R. Mato Grosso: clima - análise e representação cartográfica. Cuiabá: Entrelinhas, 2011. $102 \mathrm{p}$.

THERY, H. Routes et déboisement en Amazonie brésilienne: rondônia 1974-1996. Mappemonde, Avignon, v. 97, p. 35-40, 1997. 